\title{
EMBEDDING PRODUCTS OF CHAINABLE CONTINUA ${ }^{1}$
}

\section{RALPH BENNETT}

Bing [1] showed that every chainable continuum can be embedded in $E^{2}$. A consequence of this is that if each of $A_{1}, \cdots, A_{n}$ is a chainable continuum, then the topological product $A_{1} \times \cdots \times A_{n}$ can be embedded in $E^{2 n}$. This fact can also be derived from a theorem of Isbell [2]. This paper shows that the integer $2 n$ can be replaced by $n+1$. The following example shows it cannot be replaced by $n$.

EXAMPLE. For each integer $n$ larger than 1 the product of $n-1$ arcs and a $\sin (1 / x)$ curve cannot be embedded in $E^{n}$.

Such a continuum contains an $n$-cell and a subset disjoint from the $n$-cell with limit points in the interior of the $n$-cell.

McCord [4] has proved an elegant embedding theorem which will be the principal tool used here. He defines a map $f$ from a compact subset $X$ of a metric space $(E, d)$ to a compact subset $Y$ of $E$ to be approximable by homeomorphisms (relative to $E$ ) provided that for every $\epsilon>0$ there is an open set $U$ containing $X$ and a 1 to 1 map $\mu$ of $U$ into $E$ such that for all $x$ in $X, d(\mu(x), f(x))<\epsilon$.

Lemma (Theorem 8, Chapter IV of [4].) Let $E$ be a compact metric space and let $\left\{\left(X_{i}, f_{i}\right)\right\}$ be an inverse sequence such that each $X_{i}$ is a compact subset of $E$ and each bonding map $f_{i}$ is approximable by homeomorphisms. Then $\lim \left(X_{i}, f_{i}\right)$ can be embedded in $E$.

TheOREM. If each of $A_{1}, \cdots, A_{n}$ is a chainable continuum then $A_{1} \times \cdots \times A_{n}$ can be embedded in $E^{n+1}$.

Proof. It is known that each nondegenerate chainable continuum is homeomorphic to the inverse limit of a sequence $\left\{\left(X_{i}, f_{i}\right)\right\}$ where each $X_{i}$ is $[-1,1]$. (See, for example, [3].) If one has inverse sequences $\left(X_{i j}, f_{i j}\right), i=1, \cdots, n$, then $\prod_{i=1}^{n} \lim \left(X_{i j}, f_{i j}\right)$ is homeomorphic to $\lim \left(\prod_{i=1}^{n} X_{i j}, f_{1 j} \times \cdots \times f_{n j}\right)$ where "lim" denotes inverse limit and $\left(f_{1 j} \times \cdots \times f_{n j}\right)\left(x_{1}, \cdots, x_{n}\right)$ is always $\left(f_{1 j}\left(x_{1}\right), \cdots\right.$, $\left.f_{n j}\left(x_{n}\right)\right)$. There is an inverse sequence $\left\{\left(Y_{i}, g_{i}\right)\right\}$ such that each $Y_{i}$ is the $n$-cell $[-1,1]^{n}$, each $g_{i}$ is of the form $g_{1 i} \times \cdots \times g_{n i}$ with each $g_{j i}$ a map from $[-1,1]$ into $[-1,1]$, and such that $A_{1} \times \cdots \times A_{n}$ is homeomorphic to $\lim \left(Y_{i}, g_{i}\right)$. The inverse sequence $\left(Z_{i}, h_{i}\right)$, where

Presented to the Society, April 25, 1964; received by the editors July 2, 1964.

1 This is an improvement of a result presented to the Society, April 25, 1964. It represents a part of the author's dissertation. This work was done while the author was an N.S.F. Graduate Fellow. 
each $Z_{i}$ is $[-1,1]^{n} \times\{0\}$ and the first $n$ coordinates of $h_{i}\left(x_{1}, \cdots\right.$, $\left.x_{n}, 0\right)$ are always the first $n$ coordinates of $g_{i}\left(x_{1}, \cdots, x_{n}\right)$, has an in. verse limit homeomorphic to $A_{1} \times \cdots \times A_{n}$.

Suppose $a$ is a positive number and $i$ is a positive integer. Define $H_{i a}$ from $(-1-a, 1+a)^{n+1}$ into $E^{n+1}$ by

$$
\begin{aligned}
& H_{i a}\left(x_{1}, \cdots, x_{n}, x_{n+1}\right) \\
& \quad=\left(g_{1 i}\left(x_{1} /(1+a)\right)+\left(a x_{2} /(1+a)\right), g_{2 i}\left(x_{2} /(1+a)\right)\right. \\
& \left.\quad+\left(a x_{3} /(1+a)\right), \cdots, g_{n i}\left(x_{n} /(1+a)\right)+\left(a x_{n+1} /(1+a)\right), a x_{1}\right) .
\end{aligned}
$$

A simple calculation shows that $H_{i a}$ is a homeomorphism. For $x=\left(x_{1}, \cdots, x_{n}, 0\right)$ in $Z_{i}$ the distance from $h_{i}(x)$ to $H_{i a}(x)$ is no more than

$$
n(a /(1+a))+n \max \left\{\left|g_{j i}(b)-g_{j i}(c)\right||| b-c \mid<a /(1+a), 1 \leqq j \leqq n\right\} .
$$

If $\epsilon$ is a positive number, there is a number $a$ such that the distance from $H_{i a}(x)$ to $h_{i}(x)$ is less than $\epsilon$ for all $x$ in $Z_{i}$. That is, each $h_{i}$ is approximable by homeomorphisms. By McCord's theorem, $\lim \left(Z_{i}, h_{i}\right)$, which is homeomorphic to $A_{1} \times \cdots \times A_{n}$, can be embedded in $E^{n+1}$.

The embedding theorem of McCord was proved by constructing a sequence of continua converging to a continuum homeomorphic to the continuum he wanted to embed. The author originally proved the theorem of this paper by constructing a nested sequence of $(n+1)$ cells whose intersection was homeomorphic to the product $A_{1} \times \cdots$ $\times A_{n}$. This less elegant method gives the additional result that the embedded continuum can be assumed cellular.

The author is indebted to Michael McCord for pointing out this improved version of the author's original theorem and a major simplification of the proof. The version of the theorem of this paper presented to the Society required the assumption that each polyhedral $n$-cell in $E^{n+1}$ is collared. The author is also indebted to his major professor, W. S. Mahavier and to Lida K. Barrett for helpful discussions during the preparation of this paper.

\section{REFERENCES}

1. R. H. Bing, Snake-like continua, Duke Math J. 18 (1951), 653-663.

2. J. R. Isbell, Embeddings of inverse limits, Ann. of Math. 70 (1959), 73-84.

3. Sibe Mardešić and Jack Segal, є-mappings onto polyhedra, Trans. Amer. Math. Soc. 109 (1963), 146-164.

4. Michael McCord, Inverse limit systems, Thesis, Yale University, New Haven, Conn., 1963.

UNIVERSITY OF TENNESSEE 